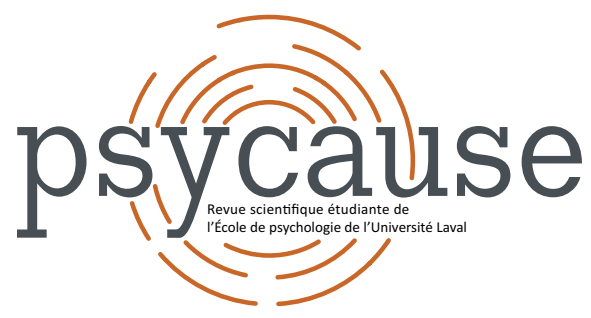

\title{
PSYCAUSE
}

Revue scientifique étudiante de l'École de psychologie de l'Université Laval

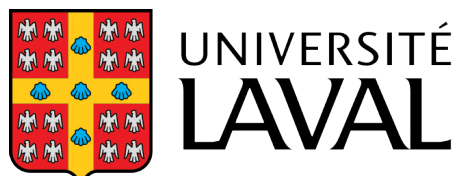

Faculté des sciences sociales École de psychologie

NOVEMBRE 2020 - VOL. $10 \mathrm{~N}^{\circ} 2$

\section{INFLUENCE DU GENRE SUR LA PERCEPTION DE L'EMPATHIE À L'AIDE D'AVATARS}

Roxanne LAVERDIÈRE' ${ }^{1}$, Lye-Ann ROBICHAUD' ${ }^{1}$, Annie TOULOUSE-FOURNIER', Audrey MARCOUX'1 \& Philip JACKSON ${ }^{1}$

${ }^{1}$ École de psychologie, Université Laval

*roxanne.laverdiere.1@ulaval.ca

\section{Pour citer l'article}

Laverdière, R., Robichaud, L.-A., Toulouse-Fournier, A., Marcoux, A. \& Jackson, P. (2020). Influence du genre sur la perception de l'empathie à l'aide d'avatars. Psycause: Revue scientifique étudiante de l'École de psychologie de l'Université Laval, 10(2), 13-15. 


\section{Discussion}

La performance diminuée des participants dans les conditions demilogue et dialogue, comparativement à celle dans la condition silence, indique que la présence de conversations en arrière-plan perturbe le fonctionnement cognitif, nuisant de ce fait à l'exécution de la tâche en cours. Cependant, contrairement à ce qui était attendu, la magnitude de l'effet de demilogue n'est pas influencée par le niveau d'impulsivité. Ainsi, en contexte de travail, les individus se rapportant comme étant plus impulsifs n'auraient pas plus de difficulté à rester concentrés sur leur tâche en présence de conversations ambiantes que ceux étant moins impulsifs. Cette observation ne corrobore pas les propositions théoriques existantes sur l'impulsivité, remettant ainsi en question l'association entre impulsivité et distractibilité.

Avant de proposer une remise en question des modèles théoriques de l'impulsivité, il est pertinent de se demander si l'absence d'effet du niveau d'impulsivité observé pourrait être attribuable, du moins en partie, aux mesures de l'impulsivité utilisées. En effet, les mesures auto-rapportées pourraient manquer de sensibilité afin d'évaluer les composantes objectives de l'impulsivité, telles que la sensibilité à la distraction. Stahl et ses collaborateurs (2014) montrent d'ailleurs que les mesures auto-rapportées ne corrèlent pas avec les mesures comportementales de l'impulsivité. L'ajout de mesures objectives complémentaires, telles que des mesures physiologiques, pourrait permettre une évaluation plus sensible des composantes comportementales de l'impulsivité, et ainsi l'émergence de conclusions différentes entre le niveau d'impulsivité et la sensibilité à la distraction.

\section{Références}

Marsh, J.E., Ljung, R., Jahncke, H., MacCutcheon, D., Pausch, F., Ball, L.J., \& Vachon F. (2018). Why are background telephone conversations distracting? Journal of Experimental Psychology: Applied, 24(2), 222-235. http://doi. org/10.1037/xap0000170

Monk, A., Fellas, E., \& Ley, E. (2004). Hearing only one side of normal and mobile phone conversations. Behaviour \& Information Technology, 23(5), 301-305. http://doi.org/10 .1080/01449290410001712744

Patton, J.H., Stanford, M.S., \& Barratt, E.S. (1995). Factor structure of the Barratt impulsiveness scale. Journal of Clinical Psychology, 51(6), 768-774. http://doi. org/10.1002/1097-4679(199511)51:6<768::AIDJCLP2270510607>3.0.CO;2-1

Stahl, C., Voss, A., Schmitz, F., Nuszbaum, M., Tüscher, O., Lieb, K., \& Klauer, K.C. (2014). Behavioral components of impulsivity. Journal of Experimental Psychology: General, 143(2), 850-886. http://doi.org/10.1037/a0033981

Whiteside, S.P., \& Lynam, D.R. (2001). The five factor model and impulsivity: Using a structural model of personality to understand impulsivity. Personality and individual differences, 30(4), 669-689. http://doi.org/10.1016/S0191$8869(00) 00064-7$

\section{Pour citer l'article}

Babin, C., Boulet, C.-A., Pépin, A. Bénard, C. \& Vachon, F. (2020). Impulsivité et distractibilité: les conversations téléphoniques en arrière-plan sont-elles particulièrement dérangeantes? Psycause: Revue scientifique étudiante de l'École de psychologie de l'Université Laval, 10(2), 11-13.

\section{INFLUENCE DU GENRE SUR LA PERCEPTION DE L'EMPATHIE À L'AIDE D'AVATARS}

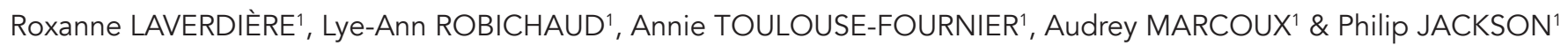

${ }^{1}$ École de psychologie, Université Laval

*roxanne.laverdiere.1@ulaval.ca

\section{Mots-clés : Empathie, genre, avatar}

L'empathie correspond à la capacité de percevoir, comprendre, se représenter, voire partager en partie les états mentaux et les sentiments d'autrui (traduction libre de Decety \& Jackson, 2004). Elle peut s'exprimer par des comportements non verbaux (p. ex., regard et inclinaison du tronc dirigés vers l'observateur, et expression faciale intéressée; Tepper
\& Haase, 1978). Ces comportements empathiques peuvent être présentés par des avatars dans les plateformes virtuelles, suscitant l'intérêt de plusieurs chercheurs en milieu clinique. L'empathie semble être influencée par plusieurs variables, dont le sexe des individus. Malgré l'absence de consensus, les données empiriques et les 
croyances populaires montrent que les femmes tendent à exprimer davantage d'empathie que les hommes (ChristovMoore et coll., 2014), notamment en raison de l'influence potentielle des rôles sociaux associés aux femmes ( $p$. ex. rôle de soignante). Les rôles sociaux et les comportements sont toutefois liés au concept du genre (Hyde, Bigler, Joel, Tate, \& van Anders, 2019), il convient donc de considérer à la fois le sexe et le genre dans l'évaluation de l'empathie. D'autres auteurs rapportent que la perception de l'empathie ne serait pas influencée significativement par le sexe de la personne observant une interaction empathique (Brugel et coll., 2015). Enfin, les données contradictoires concernant l'influence du genre et l'absence d'études utilisant des avatars pour évaluer l'empathie ne permettent pas de tirer des conclusions concernant l'influence du genre chez des avatars empathiques. L'objectif de cette étude est donc de déterminer si la moyenne d'empathie perçue varie en fonction de la représentation de l'avatar (masculin/féminin) et du genre du participant (homme/femme). En fonction des effets observés chez des humains, I'hypothèse soutenue est que les avatars féminins seront perçus plus empathiques que ceux masculins tant pour les participants s'identifiant comme une femme que ceux s'identifiant comme un homme.

\section{Méthode}

Dans le cadre de cette étude en ligne, 120 individus de la population générale, dont 44 , s'identifiant comme un homme ( $M=26,5$ ans) et $76 \mathrm{~s}^{\prime}$ identifiant comme une femme ( $M=24,6$ ans) ont complété la tâche informatique (Figure 1). Le Tableau 1 présente les données sociodémographiques collectées par le biais d'un questionnaire. Les participants ont visionné des vidéos d'avatars (deux masculins et deux féminins) affichant différents comportements non verbaux (contact visuel orienté vers le participant ou dévié; expression faciale congruente à un patient en douleur ou neutre; posture du tronc inclinée vers l'avant, centrée ou vers l'arrière). Puis, ils ont évalué l'empathie qu'ils percevaient à l'aide

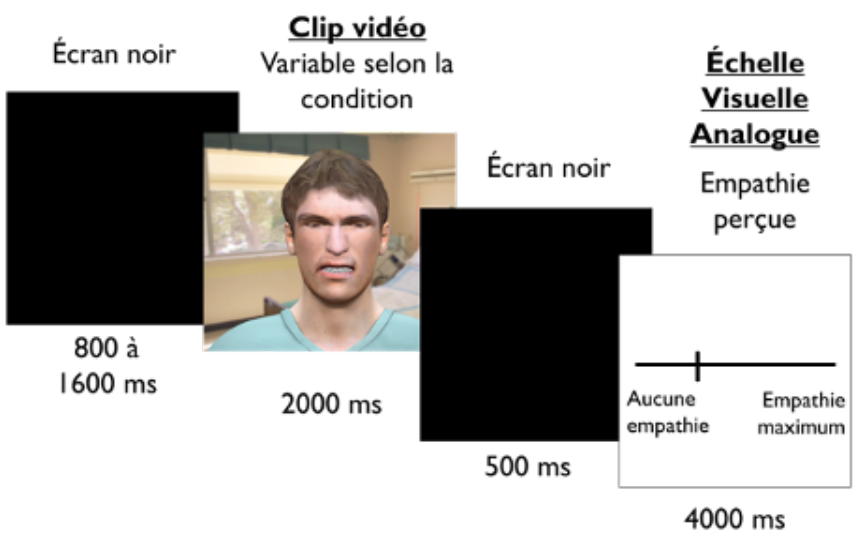

Figure 1. Séquence d'observation de la tâche informatique complétée par le participant.
Tableau 1

Données sociodémographiques des participants

\begin{tabular}{|c|c|c|}
\hline \multirow{2}{*}{ Caractéristiques } & $\begin{array}{c}\text { Genre masculina } \\
\qquad N=44\end{array}$ & $\begin{array}{c}\text { Genre féminin } \\
\quad N=76\end{array}$ \\
\hline & $\%$ & $\%$ \\
\hline \multicolumn{3}{|l|}{ Sexe biologique } \\
\hline Homme & 95,45 & 1,32 \\
\hline Femme & 4,55 & 98,68 \\
\hline \multicolumn{3}{|l|}{ Groupe culturel* } \\
\hline Canadien & 61,36 & 85,53 \\
\hline Non-Canadien & 38,64 & 14,47 \\
\hline \multicolumn{3}{|c|}{$\begin{array}{l}\text { Nombre d'heures de jeux } \\
\text { vidéo/semaine }{ }^{\star \star}\end{array}$} \\
\hline Aucune & 13,64 & 60,53 \\
\hline 1 à 8 & 63,64 & 36,84 \\
\hline 9 à 16 & 18,18 & 1,32 \\
\hline 17 à 24 & 2,27 & 1,32 \\
\hline 25 à 32 & 2,27 & 0,00 \\
\hline
\end{tabular}

* $p<0,05,{ }^{* *} p<0,001$

a Les participants de genre masculin sont âgés de 18 à 58 ans $(M=$ $26,6 ; E T=9,3)$.

${ }^{\mathrm{b}}$ Les participants de genre féminin sont âgées de 18 à 60 ans $(M=$ 24,$8 ; E T=9,5$ ).

d'une échelle visuelle analogue allant d'«aucune empathie» à «empathie maximum» (Figure 1). Pour augmenter l'engagement des participants, il était mentionné que les avatars effectuaient les mouvements et expressions faciales de réels professionnels de la santé rencontrant des patients en douleur.

\section{Résultats et discussion}

Une analyse de variance factorielle mixte 2 (avatars féminins; avatars masculins) $\times 2$ (participants de genre masculin; participants de genre féminin) a été réalisée sur la moyenne d'empathie perçue, indépendamment des comportements non verbaux affichés par les avatars. Les résultats montrent un effet principal significatif de la représentation de l'avatar, ce qui permet de confirmer l'hypothèse soutenue. L'ensemble des participants percevait davantage d'empathie exprimée par des avatars féminins $(M=43,84 ; E T=9,76)$ comparativement aux avatars masculins $(M=42,23$; $E T=$ $9,19)$. Une interprétation possible appuyée par la littérature est que les rôles sociaux généralement attribués au genre féminin (Christov-Moore et coll., 2014) pourraient aussi être attribués aux avatars féminins. Ce genre serait naturellement perçu plus chaleureux, expressif et sensible aux émotions d'autrui (Doherty et al., 1995). Les résultats montrent également que le genre du participant n'influence pas le niveau d'empathie perçue tel qu'observé par Brugel et ses collègues. (2015). Enfin, l'effet d'interaction entre la repré- 
sentation de l'avatar et le genre du participant n'est pas significatif, donc aucune analyse d'effets simples n'a été effectuée. Une interprétation possible porte sur l'hypothèse des similitudes entre les sexes (Hyde, 2005). Les hommes et les femmes seraient similaires sur plusieurs habiletés sociales (p. ex., sociabilité, communication) et certains processus cognitifs (p. ex., raisonnement abstrait, moral), ce qui pourrait se généraliser à la perception de l'empathie. Les données obtenues dans la présente étude montrent l'importance d'une représentation d'avatar féminin pour augmenter l'empathie perçue chez un observateur. Elles permettront aussi d'améliorer les plateformes virtuelles existantes, en plus d'améliorer les connaissances sur les interactions empathiques humain-machine. Dès lors, il serait pertinent d'approfondir l'apport de chaque comportement non verbal (regard, posture et expression faciale) dans la perception de l'empathie exprimée par différents avatars (féminins/ masculins) en fonction du genre du participant.

\section{Références}

Brugel, S., Postma-Nilsenova, M., \& Tates, K. (2015). The link between perception of clinical empathy and nonverbal behavior: The effect of a doctor's gaze and body orientation. Patient Education Counseling, 98(10), 1260-1265. https:// doi.org/10.1016/j.pec.2015.08.007
Christov-Moore, L., Simpson, E. A., Coude, G., Grigaityte, K., lacoboni, M., \& Ferrari, P. F. (2014). Empathy: gender effects in brain and behavior. Neuroscience \& Biobehavioral Reviews, 46 Pt 4, 604-627. https://doi.org/10.1016/j. neubiorev.2014.09.001

Decety, J., \& Jackson, P. L. (2004). The functional architecture of human empathy. Behavioral and Cognitive Neuroscience Reviews 3(2), 71-100. https://doi. org/10.1177/1534582304267187

Doherty, R. W., Orimoto, L., Singelis, T. M., Hatfield, E., \& Hebb, J. (1995). Emotional Contagion: Gender and Occupational Differences. Psychology of Women Quarterly, 19(3), 355371. https://doi.org/10.1111/j.1471-6402.1995.tb00080.x

Hyde, J. S. (2005). The gender similarities hypothesis. American Psychologist, 60(6), 581-592. https://doi. org/10.1037/0003-066X.60.6.581

Hyde, J. S., Bigler, R. S., Joel, D., Tate, C. C., \& van Anders, S. M. (2019). The future of sex and gender in psychology: Five challenges to the gender binary. American Psychologist, 74(2), 171-193. https://doi.org/10.1037/amp0000307

Tepper, D. T., \& Haase, R. F. (1978). Verbal and nonverbal communication of facilitative conditions. Journal of Counseling Psychology, 25(1), 35-44. https://doi. org/10.1037/0022-0167.25.1.35

\title{
Pour citer l'article
}

Laverdière, R., Robichaud, L.-A., Toulouse-Fournier, A., Marcoux, A. \& Jackson, P. (2020). Influence du genre sur la perception de l'empathie à l'aide d'avatars. Psycause: Revue scientifique étudiante de l'École de psychologie de l'Université Laval, 10(2), 13-15.

\section{LA RELATION ENTRE LE PERFECTIONNISME ET LES SYMPTÔMES SOMATIQUES CHEZ LES FEMMES ATTEINTES D'UN CANCER DU SEIN}

\author{
Nakita SÉGUIN-KAERCHER ${ }^{1}$, Justine CADORETTE1 ${ }^{1}$, Véronique MASSICOTE1',2,3, Hans IVERS 1,2,3 \& Josée SAVARD ${ }^{1,2,3}$ \\ ${ }^{1}$ École de psychologie, Université Laval, ${ }^{2}$ Centre de recherche du CHU de Québec-Université Laval, ${ }^{3}$ Centre de recherche \\ sur le cancer de l'Université Laval \\ *nakita.seguin-kaercher.1@ulaval.ca
}

\section{Mots-clés : Cancer, cancer du sein, perfectionnisme, symptômes somatiques}

Le cancer du sein représente le deuxième diagnostic de cancer en importance dans la population canadienne en 2020 (Société canadienne du cancer, 2020). Cette maladie et son traitement sont associés au développement de plusieurs symptômes somatiques qui peuvent affecter le fonctionnement et la qualité de vie des patientes (Ferreira et coll., 2008). Il est donc pertinent d'explorer différents facteurs qui peuvent les influencer. Parmi ceux-ci, on retrouve certains traits de personnalité, tels que le perfectionnisme (Saboonchi \& Lundh, 2003).
Les aspirations perfectionnistes correspondent à l'entretien de normes personnelles élevées, souvent irréalistes, envers soi-même. Les préoccupations perfectionnistes se manifestent, quant à elles, par une autocritique sévère, des préoccupations excessives envers le jugement des autres et des réactions négatives face à l'échec (Sirois \& Molnar, 2016).

Le perfectionnisme est associé à certains effets négatifs chez les gens souffrant de maladies chroniques (Sirois \& Molnar, 2016). Quelques données de recherches suggèrent 